\section{Unusual ocular presentations of ocular phaeohyphomycosis}

\author{
A Maudgil ${ }^{1}$, Z Johnson ${ }^{2}$, N Rogers ${ }^{1}$ \\ and HS Mudhar ${ }^{3}$
}

\begin{abstract}
igmented lesion on the conjunctiva (Figure 1a)
Abstract

Purpose Phaeohyphomycosis are melanincontaining fungi that rarely infect the eye. We describe three cases of ocular infection with some unusual clinical features.

Methods Series of three case reports describing three different presentations of phaeohyphomycosis of the eye, their histopathology, and management.

Results Case 1 mimicked an inflamed conjunctival naevus and was excised on this basis, revealing a conjunctival retention cyst containing pigmented fungal hyphae. Case 2 showed a wooden foreign body incidentally associated with pigmented fungal hyphae, which required treatment with topical

thought to be a subconjunctival haemorrhage. One month later, she returned complaining of pain and loss of pigmentation of the original lesion (Figure 1b). A second opinion diagnosed an inflamed conjunctival naevus with no intervention suggested. The patient was lost to follow-up for over a year until she presented again requesting excision of the lesion due to contact lens discomfort (Figure 1c). Excision biopsy of the conjunctiva revealed a conjunctival epithelial retention cyst containing proteinaceous, granular material (Figure 1d) with septate fungal hyphae (Figure 1e) positive for Masson Fontana (Figure 1f), diagnostic of phaeohyphomycosis.
\end{abstract} antifungal therapy. Case 3 clinically was thought to be a perforated uveal melanoma and comprised an extensive plaque of pigmented fungal hyphae over ulcerated cornea.

Conclusion The pigmented melanin containing hyphae of phaeohyphomycosis mimicked melanocytic lesions in two cases and was an incidental finding in the context of a surface foreign body.

Eye (2016) 30, 1517-1519; doi:10.1038/eye.2016.155; published online 22 July 2016

\section{Introduction}

Phaeohyphomycosis is an infection caused by melanin-containing fungi. ${ }^{1}$ The melanin is said to be a virulence factor and allows the fungus to counteract environmental oxidative stress. ${ }^{1,2}$ Phaeohyphomycosis classically causes a keratitis (especially Curvularia) and the commonest mechanism of acquiring the infection is via implantation by organic matter. ${ }^{3}$ We describe our experience of three cases; one affecting the cornea and two affecting the conjunctiva.

\section{Case reports}

Case 1

A 25-year-old female soft contact lens wearer presented acutely with a 3-week history of a

\section{Case 2}

A 9-year-old male presented after an injury to his left eye with a wooden stick 2 weeks previously. On examination, there was evidence of a partially healed conjunctival laceration with a yellowish lump visible within it, suspicious of a subconjunctival foreign body (Figure 1g). After a week of observation, excision biopsy was arranged. After the biopsy findings, G. voriconazole 1\% TDS was given, in conjunction with topical steroid cover, leading to a quiet eye (Figure 1h). The histology of the original excision showed a wooden foreign body (Figure 1i), associated with foreign body granulomatous inflammation. Brown fungi were seen on the H\&E (Figure 1j), which were positive with a Masson Fontana stain (Figure 1k), confirming phaeohyphomycosis.

\section{Case 3}

This case was referred from Pakistan to the National Specialist Ophthalmic Pathology Laboratory in Sheffield for a histological diagnosis. A 60-year-old male with a history of eye trauma 3 months previously developed a central black corneal plaque extending onto the sclera. This was suspected to be a uveal
${ }^{1}$ Department of Ophthalmology, Royal Hallamshire Hospital, Sheffield, UK

${ }^{2}$ Department of Ophthalmology, Newcastle Eye Centre, The Newcastle upon Tyne Hospitals NHS Foundation Trust, Royal Victoria Infirmary, Newcastle upon Tyne, UK

${ }^{3}$ Department of Histopathology, National Specialist Ophthalmic Pathology Service (NSOPS), Royal Hallamshire Hospital, Sheffield, UK

Correspondence: HS Mudhar, Department of Histopathology, National Specialist Ophthalmic Pathology Service (NSOPS), Royal Hallamshire Hospital, E-Floor, Glossop Road, Sheffield S10 2JF, UK Tel: +44 (0)11 42268967 ; Fax: +44 (0)1142712200. E-mail: hardeep.mudhar@ sth.nhs.uk

Received: 27 October 2015 Accepted in revised form: 6 June 2016 Published online: 22 July 2016 

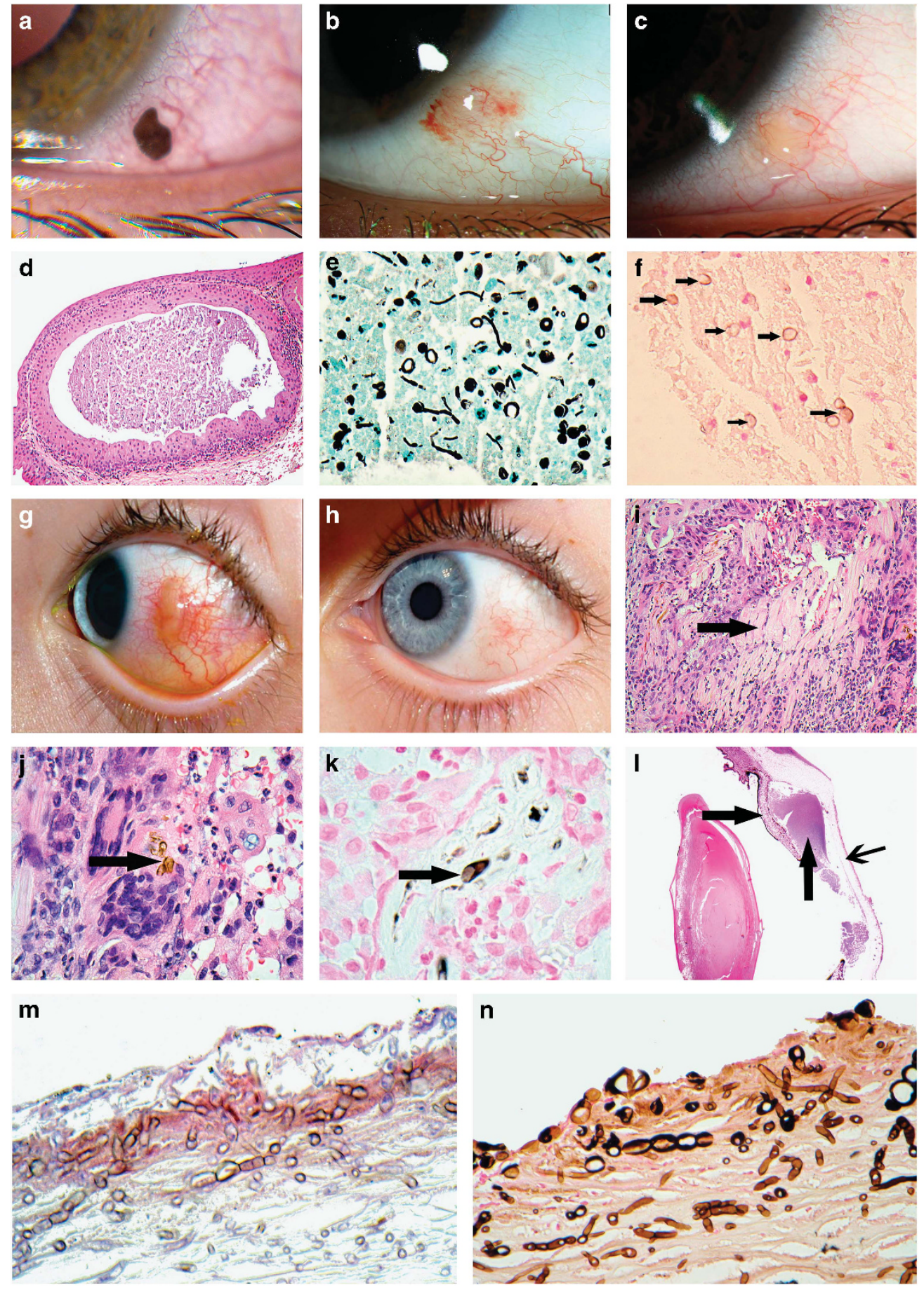

Figure 1 (a) Case 1: first clinical presentation with brown conjunctival, well-defined lesion. (b) and (c) Case 1 showing depigmentation of lesion with time. (d) haematoylin- and eosin-stained section (H\&E) of the lesion in (a) showing a retention cyst containing granular eosinophilic material. (e) silver stain of the contents of the cyst showing septate-branching fungi. (f) Masson Fontana (melanin tinctorial stain) showing brown staining of some of the fungi in the cyst (arrows). (g) Case 2 at presentation. (h) Case 2 after excision of foreign body and topical antifungal drops. (i) histology of case 2; H\&E showing the plant-based foreign body consistent with wood (arrow). (j) H\&E: the arrow points to brown fungi around the wooden foreign body. Note the giant cells in the vicinity. (k) Masson Fontana staining of Case 2 showing the presence of melanin (arrow). (l) Case 3. H\&E: enucleation specimen. The left hand arrow indicates the iris, the vertical arrow indicates pus in the anterior chamber, and the right slender arrow points to the ulcerated cornea. $(\mathrm{m}) \mathrm{H \& E}$ of the ulcerated cornea clearly showing branching, septate brown fungi. (n) Masson Fontana staining of Case 3 showing melanin. 
melanoma causing corneal perforation and the eye was enucleated. Pathological examination revealed a darkbrown corneal oval ulcer $8 \mathrm{~mm} \times 6 \mathrm{~mm}$. The latter represented a mass of brown pigmented, branching, septate hyphae of phaeohyphomycosis, associated with anterior chamber inflammation. There was no intraocular tumour (Figures 11-n).

\section{Discussion}

Phaeohyphomycosis can cause endogenous and exogenous infections including keratitis, ${ }^{2}$ endophthalmitis, ${ }^{4}$ subretinal, ${ }^{5}$ orbital, ${ }^{6}$ and periocular disease. ${ }^{7}$ In case 1 , the clinical differential diagnosis included foreign body, inflamed conjunctival naevus, and melanoma. Two previous case reports have documented pigmented fungi mimicking melanoma. The case reported by Moss et al ${ }^{8}$ involved an 85-year-old man with an enlarging conjunctival pigmented lesion, thought to be clinically a conjunctival melanoma. Excision revealed a 'dematiaceous' fungus and the patient was successfully treated with topical antifungals. In the case reported by Bui et al, ${ }^{9}$ a 75 -year-old white Caucasian woman who had sustained a traumatic tree branch injury 16 years previously developed a black cauliflower lesion over her right nasal conjunctiva. The authors were concerned it could represent a uveal melanoma with extraocular spread. However, a shave biopsy revealed a tangled mass of chromoblastomycosis.

In our case, the lesion depigmented clinically and this was reflected at the microscopic level. The melanin content of the fungal hyphae was identified with the Masson Fontana rather than seeing brown hyphae on the H\&E staining, as for cases 2 and 3. It was this depigmentation that lead to the clinical interpretation of an inflamed naevus, well known to undergo pigmentary alterations. ${ }^{10}$ Such clinical depigmentation of

phaeohyphomycosis has not been documented before in the literature. The patient was a soft contact lens wearer, which could have transmitted the fungus, (illustrated in a previous case of invasion of a soft contact lens by phaeohyphomycosis species Exophiala jeanselmei ${ }^{11}$ ), although subsequent microbiological investigations were negative.

Case 2 revealed a wooden foreign body surrounded by phaeohyphomycosis. Although intraocular ${ }^{12}$ wooden foreign bodies associated with fungi have been previously documented, this association has not been previously recorded for the conjunctiva.

Case 3's presentation as a dark-brown corneal ulcer is similar to previous case reports of keratitis caused by pigmented fungi, ${ }^{13}$ although in our case, the involvement was rather advanced; the clinical diagnosis of a perforated uveal melanoma probably reflected an unfamiliarity with intraocular melanocytic tumour presentations, as uveal melanomas rarely perforate the cornea.

Summary

What was known before

- Phaeohyphomycosis is a melanin-containing fungus. Commonly causes keratitis and ocular surface infection.

What this study adds

- Phaeohyphomycosis can mimic melanocytic neoplasms (benign and malignant). Phaeohyphomycosis can undergo depigmentation to mimic an inflammatory naevus. Plant-based conjunctival foreign bodies can lead to infection with phaeohyphomycosis.

\section{Conflict of interest}

The authors declare no conflict of interest.

\section{References}

1 Revanker SG, Sutton DA. Melanized fungi in human disease. Clin Microbiol Rev 2010; 23: 884-928.

2 Casadevall A, Rosas AL, Nosanchuk JD. Melanin and virulence in Cryptococcus neoformans. Curr Opin Microbiol 2000; 3: 354-358.

3 Wilhelmus KR, Jones DB. Curvularia keratitis. Trans Am Ophthalmol Soc 2001; 99: 111-130.

4 Kaushik S, Ram J, Chakrabarty A, Dogra MR, Brar GS, Gupta A. Curvularia lunata endophthalmitis with secondary keratitis. Am J Ophthalmol 2001; 131: 140-142.

5 Matthews BJ, Partridge D, Sheard RM, Rennie IG, Mudhar HS. A unique case of phaeohyphomycosis subretinal abscess in a patient with arthropathy and lung pathology. Indian J Ophthalmol 2013; 61(12): 763-765.

6 Kumar KK, Hallikeri K. Phaeohyphomycosis. Indian J Pathol Microbiol 2008; 51: 556-558.

7 Kerchner KR, Swing Jr DC, Williford PR. A verrucous plaque of the eyebrow. Am J Dermatopathol 2009; 31: 506-508.

8 Moss HB, Fowler WC, Schell WA, Perfect JR, Zedek DC. Dematiaceous fungi mimicking a conjunctival melanoma. Ophthal Plast Reconstr Surg 2013; 29: e65-e66.

9 Bui AQ, Espana EM, Margo CE. Chromoblastomycosis of the conjunctiva mimicking melanoma of the ciliary body. Arch Ophthalmol 2012; 130(12): 1615-1617.

10 Zamir E, Mechoulam H, Micera A, Levi-Schaffer F, Pe'er J. Inflamed juvenile conjunctival naevus: clinicopathological characterisation. Br J Ophthalmol 2002; 86(1): 28-30.

11 Hurtado I, Magran BL. Invasion of a soft contact lens by Exophiala jeanselmei. Mycopathologia 1989; 105(3): 171-173.

12 Meyer RF, Hood CI. Fungus implantation with wooden intraocular foreign bodies. Ann Ophthalmol 1977; 9(3): 271-278.

13 Tsai TH, Chen WL, Peng Y, Wang IJ, Hu FR. Dematiaceous fungal keratitis presented as a foreign body-like isolated pigmented corneal plaque: a case report. Eye 2006; 20(6): 740-741. 